08

\title{
Гетероструктуры с квантовыми точками InGaAs/GaAs, легированными атомами переходных элементов. II. Исследование циркулярно-поляризованной люминесценции
}

\author{
(C) М.В. Дорохин, ${ }^{1}$ С.В. Зайцев, ${ }^{2}$ А.В. Рыков, ${ }^{1}$ А.В. Здоровейщев, ${ }^{1}$ Е.И. Малышева, ${ }^{1}$ Ю.А. Данилов, ${ }^{1}$ \\ В.И. Зубков, ${ }^{3}$ Д.С. Фролов, ${ }^{3}$ Г.Е. Яковлев, ${ }^{3}$ А.В. Кудрин ${ }^{1}$

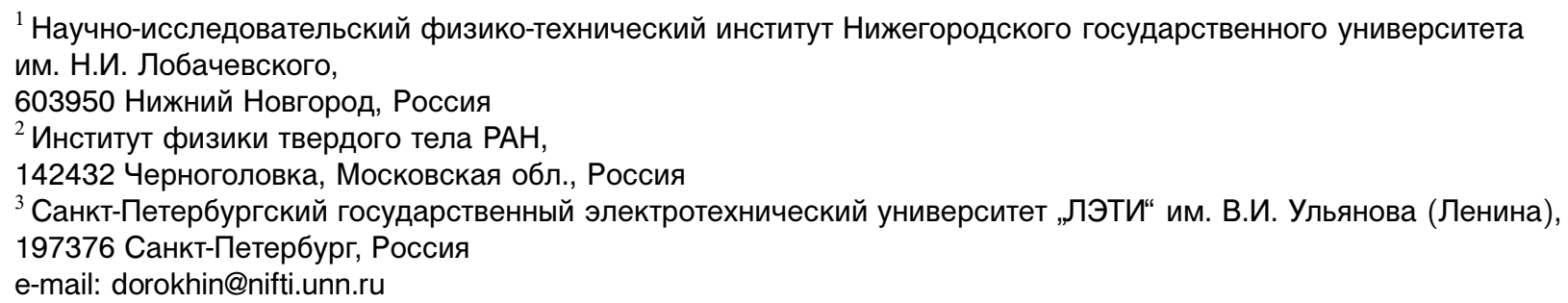

(Поступило в Редакцию 12 июля 2016 г.)

\begin{abstract}
Исследованы оптические и транспортные свойства гетероструктур с квантовыми точками InAs/GaAs, легированными атомами марганца или хрома в процессе выращивания методом MOC-гидридной эпитаксии. Показана возможность получения циркулярно-поляризованной люминесценции за счет легирования квантовых точек атомами $\mathrm{Mn}$ или $\mathrm{Cr}$, причем знак степени циркулярной поляризации зависит от вида вводимой примеси. Обнаруженный эффект объясняется особенностями излучательной рекомбинации в квантовых точках в присутствии резидентных электронов и дырок
\end{abstract}

DOI: 10.21883/JTF.2017.10.44999.1989

\section{Введение}

Легирование квантово-размерных объектов (квантовых ям [1] и квантовых точек [2,3]) атомами переходных элементов ( $\mathrm{Mn}$ или $\mathrm{Cr}$ ) является одним из приемов локальной модификации магнитных свойств материалов, который находит применение при создании полупроводниковых приборов на спин-зависимых эффектах [2,3]. В первой части настоящей работы [4] изучались квантовые точки (KT) InAs: Mn/GaAs и InAs: Cr/GaAs, сформированные методом МОС-гидридной эпитаксии. Были исследованы спектральные характеристики таких гетероструктур и показано, что варьирование концентрации $\mathrm{Mn}$ и $\mathrm{Cr}$ позволяет управлять спектральным диапазоном их люминесцентного излучения (шириной спектра, положением линий). В настоящей работе приводятся результаты исследований низкотемпературной циркулярной поляризации люминесценции указанных структур. Измерение степени циркулярной поляризации является одним из способов детектирования спинового состояния носителей заряда в полупроводниковой системе, поэтому данная методика считается одной из основных для анализа структур спинтроники [5]. Было установлено, что легирование квантовых точек атомами $\mathrm{Mn}$ или $\mathrm{Cr}$ позволяет управлять знаком циркулярнополяризованной эмиссии. Полученный эффект связывается с влиянием вида носителей заряда, вносимых легированием хромом или марганцем (электронов или дырок соответственно), на излучательную рекомбинацию с участием спин-поляризованных носителей в квантовых точках. Полученные выводы подтверждаются ре- зультатами электрохимического вольт-фарадного (ECV) профилирования и магнитотранспортных исследований.

\section{1. Методика эксперимента}

В настоящей работе рассмотрены структуры, описанные нами в [4]. Образцы для исследований представляли собой гетероструктуры с квантовыми точками, выращенные на подложке (100) n-GaAs методом газофазной эпитаксии из металлорганических соединений и арсина при атмосферном давлении газа-носителя водорода. На первой стадии выращивался буферный слой $n$-GaAs при температуре $650^{\circ} \mathrm{C}$ и тонкий $(50 \mathrm{~nm})$ слой нелегированного GaAs. Затем температура выращивания снижалась до $520^{\circ} \mathrm{C}$, и на поверхности структур формировался массив самоорганизованных квантовых точек InAs. Квантовые точки были изготовлены по стандартной методике, описанной в [6-8]. В процессе выращивания квантовых точек производилось легирование атомами $\mathrm{Mn}$ или атомами $\mathrm{Cr}$. Легирование осуществлялось методом распыления $\mathrm{Mn}(\mathrm{Cr})$ металлической мишени импульсным $\mathrm{Nd}$ : YAG лазером. Варьирования концентрации атомов $\mathrm{Mn}$ и $\mathrm{Cr}$ не выполнялось, для исследований была выбрана концентрация, обеспечивающая высокую интенсивность ФЛ в области энергий $\sim 1 \mathrm{eV}$ (указанный диапазон близок к привлекательной для волоконно-оптических линий связи длине волны $1.3 \mu \mathrm{m})$. Значение поверхностной плотности атомов марганца составило $4 \cdot 10^{12}$ и $8 \cdot 10^{12} \mathrm{~cm}^{-2}$, поверхностной плотности атомов хрома $-2 \cdot 10^{13}$ и $4 \cdot 10^{13} \mathrm{~cm}^{-2}$. После 
Параметры образцов для исследований

\begin{tabular}{c|c|c|c}
\hline Структура & $Q_{\mathrm{Mn}(\mathrm{Cr})}, \mathrm{cm}^{-2}$ & $n, \mathrm{~cm}^{-2}$ & $p, \mathrm{~cm}^{-2}$ \\
\hline $\mathrm{A} 1$ & $4 \cdot 10^{12}$ & - & - \\
$\mathrm{A} 2$ & $8 \cdot 10^{12}$ & - & - \\
$\mathrm{B} 1$ & $2 \cdot 10^{13}$ & - & - \\
$\mathrm{B} 2$ & $4 \cdot 10^{13}$ & - & - \\
$\mathrm{IA}$ & $8 \cdot 10^{12}$ & - & $1.3 \cdot 10^{10}$ \\
$\mathrm{IB}$ & $4 \cdot 10^{13}$ & $5 \cdot 10^{6}$ & - \\
$\mathrm{K}$ & 0 & - & -
\end{tabular}

Примечание. $Q_{\mathrm{Mn}(\mathrm{Cr})}-$ номинальное содержание примеси $(\mathrm{Mn}$ или $\mathrm{Cr}$ ); концентрации электронов/дырок определены из измерений эффекта Холла.

выращивания квантовых точек на поверхности формировался тонкий $(20 \mathrm{~nm})$ нелегированный покровный слой GaAs. Температура выращивания покровного слоя совпадала с температурой выращивания квантовых точек. Для определенности структуры, содержащие КТ : Mn, обозначим как структуры А, структуры с КТ : Cr - как структуры В, а контрольные структуры, не содержащие магнитной примеси в области КТ, - как структуры К.

Для исследования электрических свойств легированных марганцем (хромом) квантовых точек также были сформированы тестовые структуры (структуры IA с КТ InAs : Mn и структуры IB с KT InAs : Cr). Технология изготовления структур в точности совпадала с технологией формирования структур А и В, но эпитаксиальные слои были выращены на подложке полуизолирующего $\mathrm{GaAs}$, специального легирования эпитаксиальных слоев не выполнялось (за исключением легирования марганцем/хромом квантовых точек). Полный список и параметры образцов для исследований приведены в таблице.

Циркулярная поляризация фотолюминесцентного излучения в магнитном поле исследовалась в криостате со сверхпроводящим магнитом под откачкой в сверхтекучем гелии (температура $T \approx 2 \mathrm{~K}$ ) в геометрии Фарадея (магнитное поле $B=0-3 \mathrm{~T}$ направлено по нормали к поверхности образцов). Измерения выполнялись по стандартной методике с четвертьволновой пластиной и поляризатором [5]. Для возбуждения циркулярнополяризованной люминесценции использовался Не- $\mathrm{Ne}-$ лазер. Значение степени циркулярной поляризации рассчитывалось по формуле

$$
P_{P L}=\left(I^{+}-I^{-}\right) /\left(I^{+}+I^{-}\right),
$$

где $I^{+}\left(I^{-}\right)$- интенсивности компонент с левой (правой) поляризацией, полученные путем интегрирования полосы соответствующей части спектра излучения. Для исследованных структур интегрировалась спектральная линия, соответствующая излучению с энергией $\sim 0.95 \mathrm{eV}$ для InAs: Mn КТ и $\sim 1.05 \mathrm{eV}$ для InAs : Cr КТ (вставки на рис. 1, $a$ и $b$ ).

Для определения степени легирования квантовых точек атомами марганца использовался метод электрохимического вольт-фарадного профилиро- вания (ECV). Измерения производились на установке ECVPro (Nanometrics) $[9,10]$ с применением водного раствора $0.1 \mathrm{M}$ тайрона (tiron) в качестве электролита. Из-за большой приповерхностной концентрации примеси использовалась низкая частота измерения $(93 \mathrm{~Hz})$. Напряжение смещения при измерении емкости выбиралось таким, чтобы ширина области объемного заряда (OO3) барьера электролит-полупроводник была минимальной, a $\operatorname{tg} \delta$ был меньше 0.4 .

Дополнительно измерение концентрации носителей заряда в структурах с квантовыми точками (в случае ее низкого значения) выполнялось методом Ван дер Пау (для этого формировались 4 омических контакта). Исследования проводились для контрольных структур IA, IB на автоматизированной установке Nanometrics HL5500PC Hall Effect Measurement System в диапазоне температур 77-300 K.
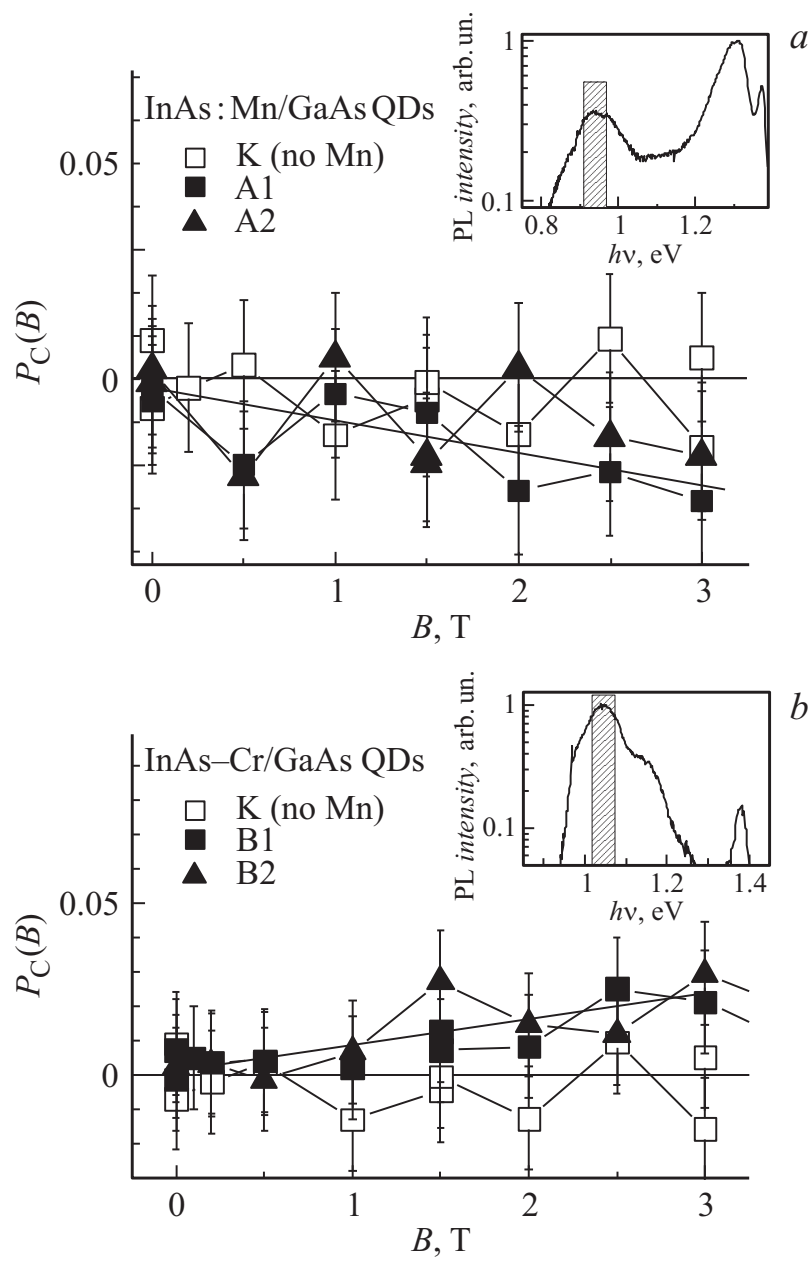

Рис. 1. Магнитополевая зависимость степени циркулярной поляризации фотолюминесценции $P_{C}(B)$ структур с КТ InAs : Mn $(a)$ и InAs : $\mathrm{Cr}(b)$, измеренная при температуре $2 \mathrm{~K}$. Повторные точки $P_{C}(B)$ для контрольной структуры $\mathrm{K}$ при магнитном поле $B=0,1.5$ и 3 Т демонстрируют методическую экспериментальную ошибку $( \pm 1.5 \%)$. На вставках показаны спектры ФЛ структур $(T=2 \mathrm{~K})$, на спектрах выделена область интегрирования для расчета $P_{C}$ по формуле (1). 


\section{2. Экспериментальные результаты}

\section{1. Магнитолюминесценция структур, легированных марганцем и хромом}

На рис. 1 представлены магнитополевые зависимости степени циркулярной поляризации для структур А (рис. $1, a)$ и В (рис. $1, b)$ с КТ, легированными марганцем и хромом соответственно. В магнитном поле излучение исследованных структур становится частично циркулярно-поляризованным, величина степени циркулярной поляризации по абсолютному значению превышает ошибку измерений $(\sim 0.015)$, полученную из измерения контрольной структуры К (для которой значение степени циркулярной поляризации в использованном диапазоне магнитных полей близко к нулю). Следует подчеркнуть зарегистрированное различие в знаках степени циркулярной поляризации. Для структур, легированных атомами Mn (с концентрацией 4 и $\left.8 \cdot 10^{12} \mathrm{~cm}^{-2}\right)$, значение степени циркулярной поляризации отрицательное, т. е. интенсивность $\sigma^{-}$-компоненты выше интенсивности $\sigma^{+}$-компоненты ФЛ излучения при энергии $1 \mathrm{eV}$. Для обеих исследованных структур В, легированных $\mathrm{Cr}$ (с концентрацией 2 и $4 \cdot 10^{13} \mathrm{~cm}^{-2}$ ), значение степени циркулярной поляризации положительное (т.е. интенсивность $\sigma^{+}$-компоненты выше интенсивности $\sigma^{-}$-компоненты). Различие между зависимостями для разных концентраций $\mathrm{Mn}$ и $\mathrm{Cr}$ (квадраты и треугольники на рис. 1) не может быть установлено в силу относительно высокого значения погрешности измерений.

Известно, что легирование полупроводника атомами переходных металлов (таких, как $\mathrm{Mn}, \mathrm{Ni}, \mathrm{Co}$ или $\mathrm{Cr}$ ) приводит к существенному изменению его люминесцентных свойств, таких как циркулярная поляризация рекомбинационного излучения в магнитном поле, появлению сильной магнитополевой зависимости энергий оптических переходов (изменение $g$-фактора носителей заряда), а также модифицирует когерентные свойства электронной спиновой системы [11]. Применительно к квантовым точкам наличие резидентных носителей (электронов или дырок) изменяет структуру энергетических уровней нейтральных и заряженных комплексов (экситонов и трионов) в КТ в их основном и возбужденном состояниях, аналогично случаю квантовых ям [6]. Такое изменение, в свою очередь, должно приводить к изменению знака циркулярной поляризации люминесценции $P_{C}(B)$ в магнитном поле $B>0$ в зависимости от знака заряда носителей ( $n$-тип или $p$-тип проводимости системы).

Наблюдаемое различие объясняется разницей в структуре энергетических уровней фотовозбужденного экситона, захваченного и локализованного в положительно или отрицательно заряженной КТ (рис. 2). Так, при общей не очень высокой концентрации Mn в случае одиночного атома Мn в самой КТ или рядом, он замещает катион в матрице полупроводника $A^{3} B^{5}$ и отдает одну дырку в КТ. В этом случае основным состоянием КТ в ненулевом магнитном поле будет расщепленное по квантовому числу $m_{j}= \pm 3 / 2$ состояние дырки с $g$-фактором $g_{h}$ (рис. $2, b$ ). При фотовозбуждении экситона нижайшим по энергии состоянием системы будет синглет, когда две дырки (резидентная дырка и дырка, входящая в состав захваченного в КТ экситона) имеют противоположный спин. Расщепление энергии в магнитном поле этого возбужденного состояния системы будет определяться $g$-фактором оставшегося неспаренным электрона $\left(g_{e}\right)$ со спином $\pm 1 / 2$. На рис. 2 учтено, что в системе InAs-GaAs оба $g$-фактора отрицательны: $g_{e}$, $g_{h}<0$, а также $\left|g_{e}\right|>\left|g_{h}\right|[12]$. В начальный момент при линейно поляризованном фотовозбуждении рождается одинаковое количество экситонов, соответствующих обеим циркулярным поляризациям $\sigma^{+}$и $\sigma^{-}$и имеющим электронный спин $\pm 1 / 2$, которые захватываются в КТ, образуя положительно заряженный экситон (трион). Такой положительный трион к моменту рекомбинации может релаксировать в нижайшее по энергии состояние со спином $+1 / 2$ (при $\left.g_{e}<0\right)$, соответствующее компоненте с циркулярной поляризацией $\sigma^{-}$(рис. 2, $b$ ), поэтому в излучательной рекомбинации должна преобладать циркулярная поляризация $\sigma^{-}$, в меру различия в заполнении состояний электронных уровней триона.

Величина $P_{C}(B)$ определяется соотношением времен рекомбинации и спиновой релаксации электрона, поэтому отсутствие в эксперименте заметной циркулярной поляризации в КТ, легированных атомами $\mathrm{Mn}$, свидетельствует о слабой спиновой релаксации электронов. Это согласуется с длительными временами спиновой релаксации электронов $(\sim 5-10 \mathrm{~ns})$ в силу полного пространственного квантования носителей заряда в КТ, что значительно превышает времена рекомбинации нейтральных экситонов и трионов ( 1-2ns) [13]. Кроме того, не наблюдается заметного сдвига $(>1 \mathrm{meV})$ энергий оптических переходов в КТ, характерного для $s, p$ - $d$ обменного взаимодействия с магнитными ионами переходного металла при их заметной концентрации $\sim 0.1-10 \%$, в первую очередь, такого иона, как $\mathrm{Mn}$ [12]. В случае же одиночного атома Mn в КТ его обменное взаимодействие с экситоном приводит к возникновению тонкой структуры энергетических уровней последнего с расщеплением 100-200 $\mu \mathrm{V}$ [15].

Для структур $\mathrm{B}$, легированных атомами $\mathrm{Cr}$, конечные положительные значения $P_{C}(B)$ (рис. $\left.1, b\right)$ аналогичны случаю дырок в KT InAs : Mn, объясняются наличием равновесных электронов в системе. В этом случае наличие резидентного электрона в КТ качественно изменяет картину энергетических уровней такой отрицательно заряженной КТ. Основным состоянием КТ в магнитном поле будет расщепленное по спину состояние электрона, а возбужденным состоянием системы будет синглет с двумя спаренными электронами и неспаренной дыркой с моментом $\pm 3 / 2$ - отрицательно заряженный трион (рис. 2,c). Спиновая релаксация дырки в КТ хоть и ослаблена ее полным пространственным квантованием 


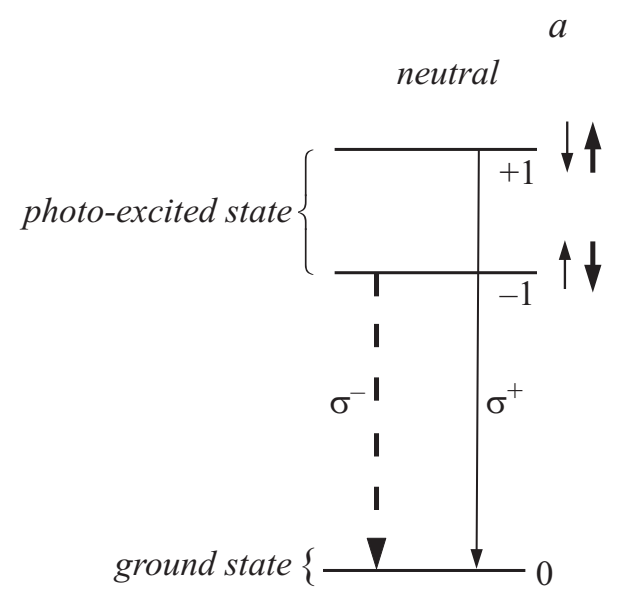

$b$

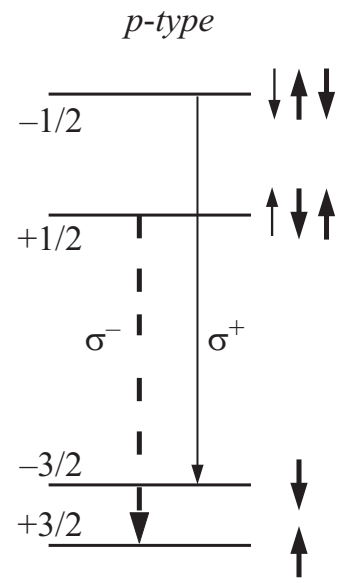

C

n-type

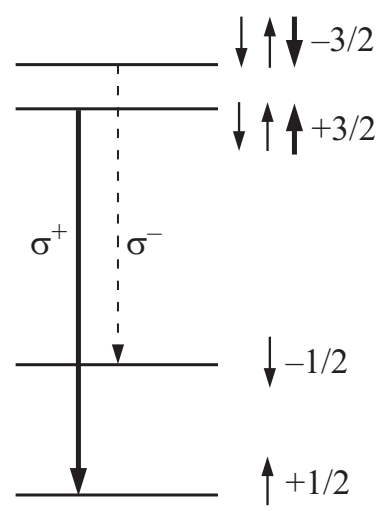

Рис. 2. Схема энергетических уровней системы электронов и дырок для основного и возбужденного состояний, а также оптических переходов в нейтрально $(a)$, положительно $(b)$ и отрицательно $(c)$ заряженной КТ во внешнем магнитном поле. Более толстая стрелка маркирует более вероятный оптический переход. Короткие тонкие и толстые стрелки обозначают электроны и дырки соответственно. На рисунке учтено, что $g$-факторы обоих носителей отрицательны, как в системе InAs-GaAs [12].

тем не менее в силу сложного характера валентной зоны обычно происходит быстрее, чем релаксация спина электрона, и сокращается до времен, сравнимых со временами излучательной рекомбинации $[7,13]$. Аналогично случаю КT InAs : Mn за время жизни отрицательного триона также возникает разность населенностей дырочных уровней в пользу дырок с моментом $+3 / 2$, соответствующих $\sigma^{+}$циркулярной поляризации ФЛ, в согласии с экспериментом $P_{C}(B)>0$ (рис. $\left.1, b\right)$. Подчеркнем, что в предположении о нейтральности структур с КТ, легированных атомами $\mathrm{Cr}$, схема излучательных переходов будет иметь вид, как показано на рис. 2, $a$, аналогичный случаю квантовых ям [6]. Согласно рис. 2, $a$, в указанной схеме наиболее вероятны переходы, обусловливающие отрицательный знак $P_{C}(B)$, следовательно, регистрация положительного знака $P_{C}(B)$ может быть объяснена только наличием в квантовых точках резидентных электронов.

\section{2. Измерение концентрации носителей заряда в массивах квантовых точек, легированных атомами $\mathrm{Mn}$ или $\mathrm{Cr}$}

Для подтверждения модели излучательной рекомбинации (рис. 2) и факта наличия резидентных дырок в структурах с КТ: Mn и электронов в КТ: $\mathrm{Cr}$ были выполнены эксперименты по измерению концентрации носителей заряда в структурах IA и IB. На рис. 3 показаны профили концентрации основных носителей заряда в приповерхностной области структуры IA (с КТ, легированными $\mathrm{Mn}$ ), а также контрольной нелегированной структуры, рассчитанные по электрохимическим вольтфарадным измерениям. Анализ контрольной структуры показал дырочную проводимость с максимумом концентрации $\left(\sim 3 \cdot 10^{18} \mathrm{~cm}^{-3}\right)$ на глубине $24 \mathrm{~nm}$ от поверхно-

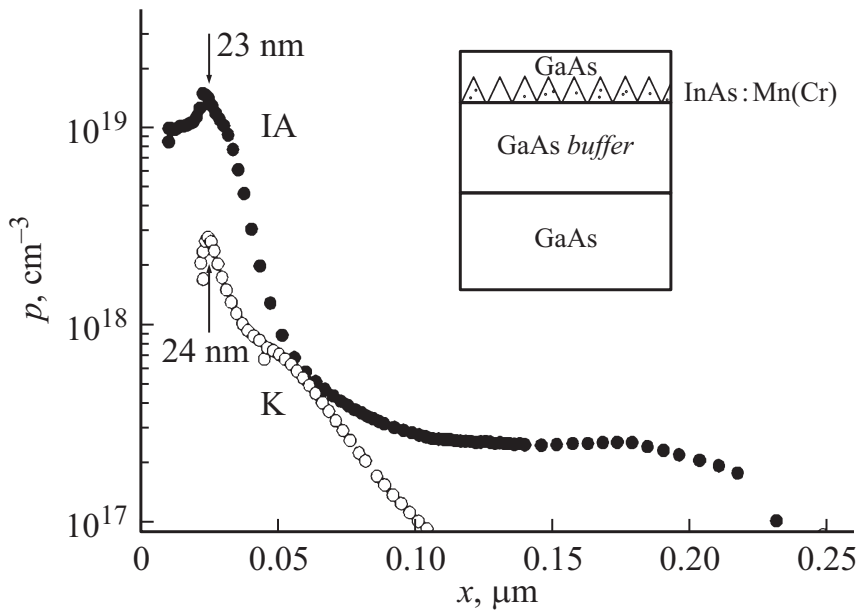

Рис. 3. Профили распределения концентрации основных носителей заряда, определенные методом электрохимического вольт-фарадного профилирования, для структур с КT InAs : Mn (IA) и контрольной нелегированной структуры с КТ (K). На вставке приведено схематическое изображение структур для исследований.

сти. Данное расстояние от поверхности приблизительно соответствует положению слоя квантовых точек в структуре. Введение марганца в область квантовых точек приводит к увеличению общей концентрации дырок, в том числе концентрации в максимуме (по-видимому, в области КТ) до величины $\sim 1.5 \cdot 10^{19} \mathrm{~cm}^{-3}$ (это объясняется акцепторной природой примеси $\mathrm{Mn}$ в полупроводниках $\left.A^{3} B^{5}[8]\right)$. Для структуры IA положение максимума несколько отличается от предыдущего случая и составляет $23 \mathrm{~nm}$. Полученные значения укладываются в погрешность измерений и технологический разброс параметров структур и, таким образом, также могут 


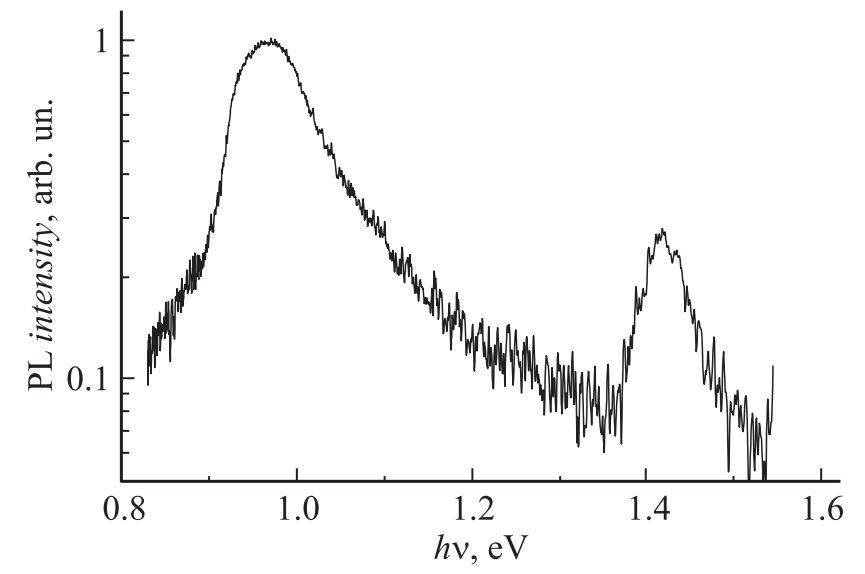

Рис. 4. Спектр фотолюминесценции структуры IA, измеренный при температуре $300 \mathrm{~K}$.

быть сопоставлены с положением квантовых точек в структуре. Наличие дырок в приповерхностной области контрольной структуры К обусловлено фоновым легированием. Фоновое легирование, придающее эпитаксиальным слоям дырочную проводимость, является характерной особенностью МОС-гидридной эпитаксии (см., например, [16]).

Результаты электрохимического вольт-фарадного профилирования структур IA могут быть дополнены измерениями эффекта Холла. Результаты измерения исследованных структур приведены в таблице. Для структур с номинальной концентрацией $\mathrm{Mn}$, равной $8 \cdot 10^{12} \mathrm{~cm}^{-2}$, была зарегистрирована дырочная проводимость с поверхностной плотностью носителей заряда, равной $1.3 \cdot 10^{10} \mathrm{~cm}^{-2}$. Полученные значения поверхностной плотности ниже, чем усредненное по глубине $0.25 \mu \mathrm{m}$ (суммарная толщина эпитаксиальных слоев) значение объемной концентрации, рассчитанной по электрохимическим измерениям $\left(10^{12} \mathrm{~cm}^{-2}\right)$. Это свидетельствует о том, что даже при комнатной температуре большая часть дырок локализована в области квантовых точек и не участвует в процессах проводимости (и соответственно не дает вклада в коэффициент Холла). Локализация носителей в КТ подтверждается спектрами ФЛ, измеренными при комнатной температуре (рис. 4). На спектре наблюдается широкая полоса с максимумом при энергии $\sim 0.97 \mathrm{eV}$, соответствующая излучательным переходам в KT InAs [17]. Интенсивность линий КТ выше, чем интенсивность фундаментальной полосы ФЛ $\mathrm{GaAs}$ при $\sim 1.42 \mathrm{eV}$, что согласуется с предположением о локализации носителей заряда в КТ. Локализация дырок в КТ при комнатной температуре для структур, аналогичных исследованным, была также подтверждена в [18].

Для структур IB c KT InAs : Cr использование метода ECV оказывается затруднительным в силу компенсирующих свойств примеси $\mathrm{Cr}$. В отличие от марганца введение хрома приводит к снижению концентрации дырок, регистрируемых в системе за счет фонового легирования [19]. Уменьшение концентрации дырок ведет к увеличению толщины обедненного слоя, что делает невозможным измерение методом ECV концентрации основных носителей заряда в приповерхностной области. Измерения вольт-фарадных характеристик структур IB методом ECV не позволили определить концентрацию основных носителей заряда в области КТ, что является косвенным подтверждением изолирующих свойств материала с КТ, легированными хромом. Поэтому для оценки типа носителей заряда в KT InAs : Cr были использованы измерения эффекта Холла. Значение концентрации электронов, полученное для структуры IB, приведено в таблице.

Согласно результатам измерений, в структурах с КТ, легированных хромом, была зарегистрирована электронная проводимость с поверхностной концентрацией порядка $5 \cdot 10^{6} \mathrm{~cm}^{-2}$. Полученные результаты объясняются природой примесного центра атома $\mathrm{Cr}$, являющегося глубокой донорной примесью в InAs [19]. Введение хрома, по-видимому, привело не только к компенсации фонового легирования полупроводниковых слоев, но также к появлению электронной проводимости, однако со сравнительно высоким слоевым сопротивлением $\left(\sim 10^{9} \Omega / \square\right)$. Отметим, что при температуре жидкого азота $(77 \mathrm{~K})$ измерение эффекта Холла на структурах становится невозможным. Кроме этого, оценка концентрации электронов из измерений структур IB, выполненных при $300 \mathrm{~K}$, также затруднительна из-за высокого значения слоевого сопротивления, сопоставимого с сопротивлением полупроводниковой подложки. В то же время полученные данные косвенно подтверждают наличие резидентных электронов в КT InAs : Cr.

Таким образом, исследования эффекта Холла и электрохимическое вольт-фарадное профилирование структур с KT InAs : Mn показали наличие в КТ резидентных дырок, что согласуется с результатами исследований циркулярно-поляризованной фотолюминесценции.

Измерение электрических характеристик структур с KT InAs: Cr косвенно подтверждает наличие в КТ резидентных электронов, что предполагалось при объяснении циркулярно-поляризованной ФЛ. Отметим, что как наличие дырок в InAs KT, так и отсутствие резидентных носителей заряда, согласно схемам излучательных переходов (рис. 2,a), обусловливают отрицательный знак поляризации $[12,13]$. Наличие положительной циркулярной поляризации со степенью выше порога обнаружения может быть объяснено наличием в КТ резидентных электронов. Таким образом, измерение знака циркулярной поляризации магнитолюминесценции позволяет независимо и бесконтактно определить знак заряда носителей в КТ при низких температурах в структурах, для которых значение концентрации носителей заряда лежит вблизи предела обнаружения. 


\section{3. Заключение}

В настоящей работе проведено исследование циркулярно-поляризованной фотолюминесценции гетероструктур с KT InAs/GaAs, легированными атомами марганца или хрома в процессе выращивания. Введение примеси переходных элементов позволяет управлять типом проводимости квантовых точек: вносит дополнительные дырки при легировании марганцем и формирует изолирующий слой КТ с низкой концентрацией электронов при легировании атомами хрома. Показано, что изменение типа проводимости позволяет изменять знак циркулярно-поляризованной фотолюминесценции в структурах с КТ. В то же время знак степени циркулярной поляризации является индикатором типа проводимости в исследованных структурах.

Авторы выражают благодарность Б.Н. Звонкову за формирование образцов для исследований. Работа выполнена в рамках реализации государственного задания (проекты № 8.1054.2014/К, 3.285.2014/K, 3423, 16.1307.2014К) Минобрнауки России, при поддержке РФФИ (гранты № 15-02-07824, 15-38-20642мол_а_вед и 16-07-01102), а также гранта президента РФ (МК$8221.2016 .2)$.

\section{Список литературы}

[1] Poggio M., Myers R.C., Stern N.P., Gossard A.C, Awschalom D.D // Phys. Rev. B. 2005. Vol. 72. P. 235313.

[2] Буравлев А.Д., Неведомский В.Н., Убыйвовк Е.В., Сапега В.Ф., Хребтов А.И., Самсоненко Ю.Б., Цырлин Г.Э., Устинов В.М. ФТП. 2013. Vol. 47. N 8. P. 1033.

[3] Holub M., Chakrabarti S., Fathpour S., Bhattacharya P., Lei Y., Ghosh S. Appl. Phys. Lett. 2004. Vol. 85. N 6. P. 973.

[4] Дорохин М.В., Здоровейщев А.В., Малымева Е.И., Данилов Ю.А., Звонков Б.Н. // ФТП. 2016.

[5] Holub M., Bhattacharya P. // Phys. D: Appl. Phys. 2007. Vol. 40. R179.

[6] Bartsch G., Gerbracht M., Yakovlev D.R., Blokland J.H., Christianen P.C.M., Zhukov E.A., Dzyubenko A.B., Karczewski G., Wojtowicz T., Kossut J., Maan J.C., Bayer M. // Phys. Rev. B. 2011. Vol. 83. P. 235317.

[7] Gundogdu K., Hall K.C., Koerperick E.J., Pryor C.E., Flatte M.E., Boggess Thomas F., Shchekin O.D., Deppe D.G. // Appl. Phys. Lett. 2005. Vol. 86. P. 113111.

[8] Ilegems M., Digle R., Rupp L.W.Jr. // J. Appl. Phys. 1975. Vol. 46. N 7. P. 3059.

[9] Zubkov V., Kucherova O., Frolov D., Zubkova A. // Phys. St. Sol. C. 2013. Vol. 10. N 3. P. 342.

[10] Яковлев Г.Я., Фролов Д.С., Зубкова А.В., Левина А.А., Зубков В.И., Соломонов А.В., Стерлядкин О.К., Сорокин C.A. // ФТП. 2016. Т. 50. № 3. С. 324.

[11] Yakovlev D.R., Bayer M. Spin Physics in Semiconductors / Ed. by M.I. Dyakonov / Berlin: Springer, 2008. Chap. 6.

[12] Wimbauer Th., Oettinger K., Efros Al.L., Meyer B.K., Brugger H. // Phys. Rev. B. 1994. Vol. 50. P. 8889.

[13] Marie X., Urbaszek B., Krebs O., Amand T. Spin Physics in Semiconductors / Ed. by M.I. Dyakonov. Berlin: Springer, 2008. Chap. 4.
[14] Cibert J., Scalbert D. Spin Physics in Semiconductors / Ed. by M.I. Dyakonov. Berlin: Springer, 2008. Chap. 13.

[15] Kudelski A., Lemaitre A., Miard A., Voisin P., Graham T.C.M., Warburton R.J., Krebs O. // Phys. Rev. Lett. 2007. Vol. 99. P. 247209.

[16] Doi A., Aoyagi Y., Namba S. // Appl. Phys. Lett. 1986. Vol. 48. N 26. P. 1787.

[17] Автореф. канд. дис. Здоровейщев А.В. Н. Новгород. 2006. $22 \mathrm{c}$.

[18] Vasilevskiy M.I, Baidus N.V., Cavaco A., Sobolev N.A., Carmo M.C., Alves E., Zvonkov B.N. // J. Appl. Phys. 2008. Vol. 103. N 8. P. 083548.

[19] Zucca R. // J. Appl. Phys. 1977. Vol. 48. N 5. P. 1987. 